\title{
A case of fatal gastrointestinal hemorrhage in granulomatosis with polyangiitis
}

\author{
Siew May Wang*1, Ai-Ping Chua ${ }^{1}$, Chamara P. Ranasinghe ${ }^{1}$, Yin Huei Pang ${ }^{2}$, Jingxiang Huang ${ }^{2}$, Kenneth Koo ${ }^{1}$ \\ ${ }^{1}$ Department of Medicine, Ng Teng Fong General Hospital, Singapore \\ ${ }^{2}$ Department of Pathology, National University Hospital, Singapore
}

Received: February 20, 2017

DOI: $10.5430 /$ crim.v4n2p34
Accepted: March 20, 2017

Online Published: March 31, 2017

\begin{abstract}
Although granulomatosis with polyangiitis (GPA) can affect any organ, gastrointestinal (GI) involvement is uncommon. Moreover, death from massive GI hemorrhage is a rare entity, with few cases described in the literature. In our report, we present a case of a patient with GPA who developed massive ulcerative bleeding, which ultimately proved fatal. Given the paucity of reports available, the significant potential for morbidity, and the fact that concurrent immunosuppressive therapy may themselves exacerbate the risk of bleeding, we reviewed 49 case reports of patients with GPA and GI involvement from 1982 to June 2016 in an attempt to shed light on a little seen sequelae that warrants a high index of suspicion.
\end{abstract}

Key Words: Granulomatosis with polyangiitis, Gastrointestinal hemorrhage, Immunosuppressive therapy

\section{INTRODUCTION}

Granulomatosis with polyangiitis (GPA) is a systemic vasculitis of small- and medium-sized vessels characterized by granulomatous and necrotizing inflammation with the potential for multi-organ involvement, most commonly affecting the respiratory tract and kidneys. ${ }^{[1]}$ Gastronintestinal (GI) manifestations in GPA are uncommon, but the morbidity from GI involvement can be severe. ${ }^{[2]}$

In our case report, we describe an unusual case of GPA that presented with catastrophic upper GI ulcers that proved fatal. Our report also reviews the relevant literature, including 49 case reports of GPA involving the GI tract, published from 1982 to June 2016, in an attempt to amalgamate the most common clinical manifestations and management approaches of this little-seen entity.

\section{Case PResentation}

In January of 2015, a 61-year-old gentleman, Mr. X, presented to our hospital with a five-day history of persistent abdominal pain, with associated anorexia and weight loss. Despite an unremarkable abdominal examination, there were concerns for malignancy, and thus an abdominal and pelvic computed tomography (CT) was performed. Imaging failed to reveal any intra-abdominal or intra-pelvic abnormalities, but did note an irregular mass in the lower lobe of the right lung. A subsequent CT thorax confirmed the presence of a mass in the right lung, measuring $5.4 \mathrm{~cm} \times 4.6 \mathrm{~cm}$, in addition to another irregular mass in the upper lobe of the left lung, measuring $6.0 \mathrm{~cm} \times 5.4 \mathrm{~cm}$ encasing the origin of the left subclavian artery.

Histology obtained via a transthoracic lung needle biopsy demonstrated extensive necrosis with suppuration, fibrinous

*Correspondence: Siew May Wang, MBChB; Email: siewmay2020@ hotmail.com; Address: 2500 Metrohealth Dr, Cleveland, Ohio, 44109, USA. 
exudate, and focal areas of regenerative stromal tissue with no evidence of malignant cells, vasculitis, or well-formed granulomas.

After defaulting on follow-up, Mr. X re-presented one month later following a bout of hemoptysis. On this occasion, a repeat CT thorax showed interval progression of the initial lung with an increase in size and mediastinal extension. He would undergo a bronchoscopy and a transtracheal needle biopsy of the right lung lesion, which again demonstrated necrotic tissue with scattered multinucleate giant cells and fibrosis. Of note, all fungal and bacterial respiratory cultures had returned negative at this point.

Two weeks following his second lung biopsy, Mr. X began to develop purpuric skin lesions and gingivitis associated with an acute kidney injury and normocytic normochromic anemia. An autoimmune screen was positive for c-ANCA. He would eventually undergo skin and gum biopsies showing medium-sized vasculitis with granuloma (see Figure 1 and Figure 2), and a kidney biopsy revealing ANCA-associated segmental necrotizing glomerulonephritis with crescents (see Figure 3). Mr. X was ultimately diagnosed with GPA.

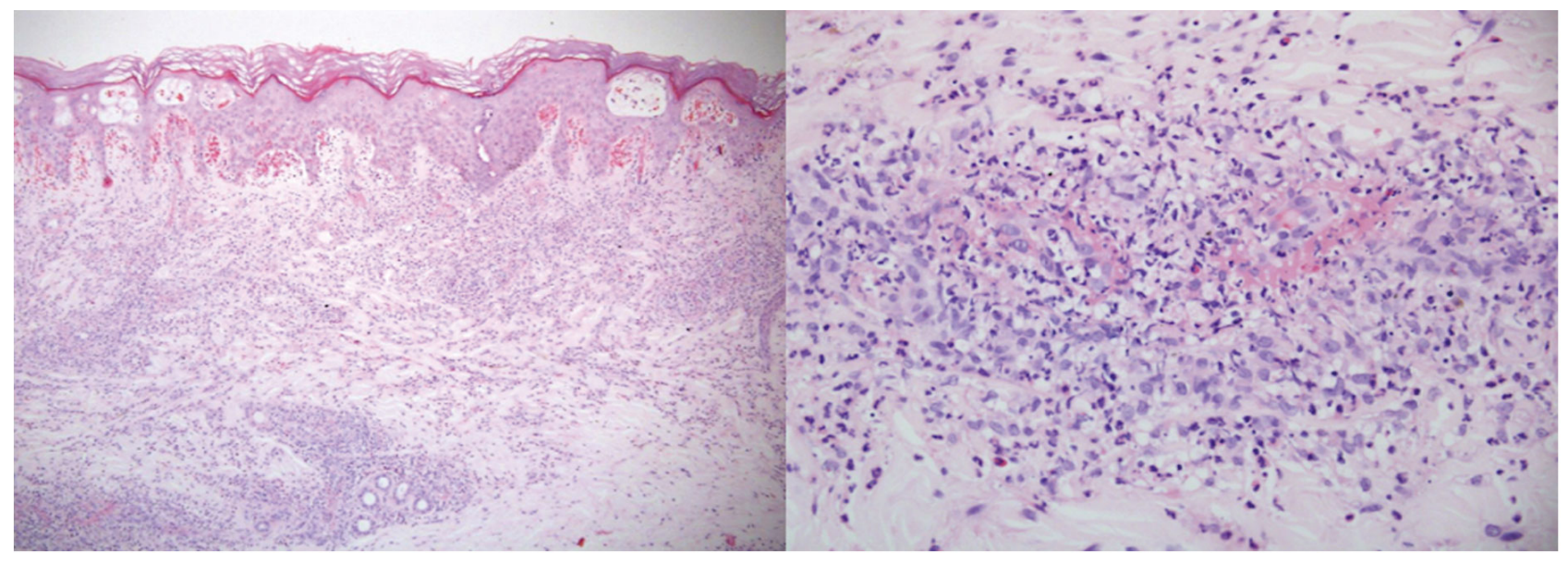

Figure 1. Skin biopsy. Hematoxylin \& eosin (H\&E) stain $(40 \times[$ left] and 400× [right] magnification): Leukocytoclastic vasculitis with fibrinoid necrosis, neutrophilic infiltrate and karryorhectic debris.

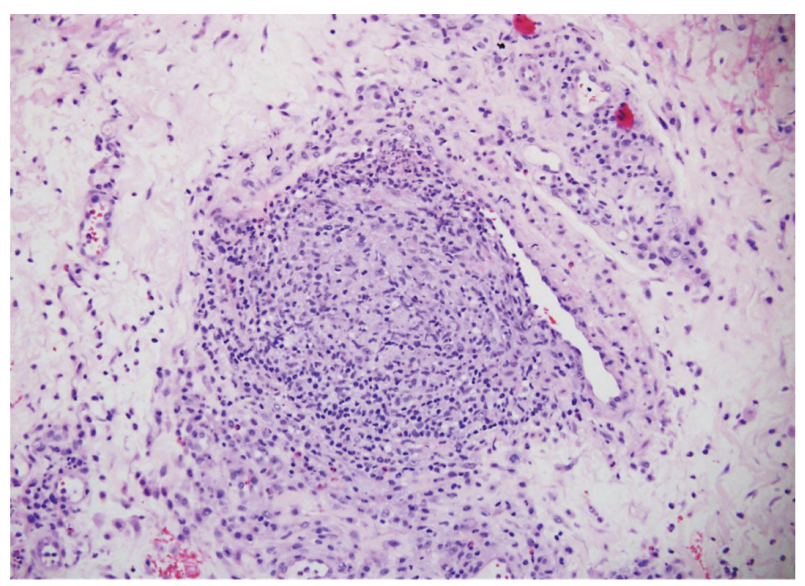

Figure 2. Oral mucosa biopsy. Hematoxylin \& eosin (H\&E) stain $(200 \times$ magnification): Granulomatous vasculitis.

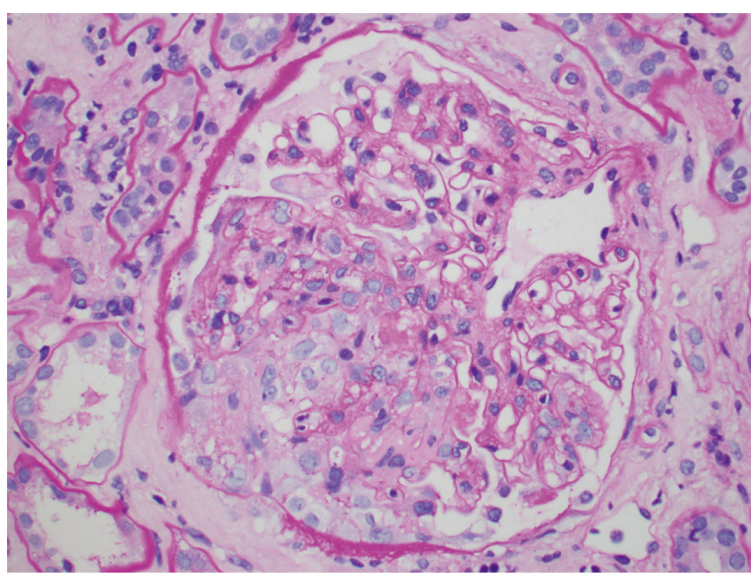

Figure 3. Kidney Biopsy. Periodic acid-Schiff (PAS) stain (400× magnification): Glomeruli with cellular crescent. 

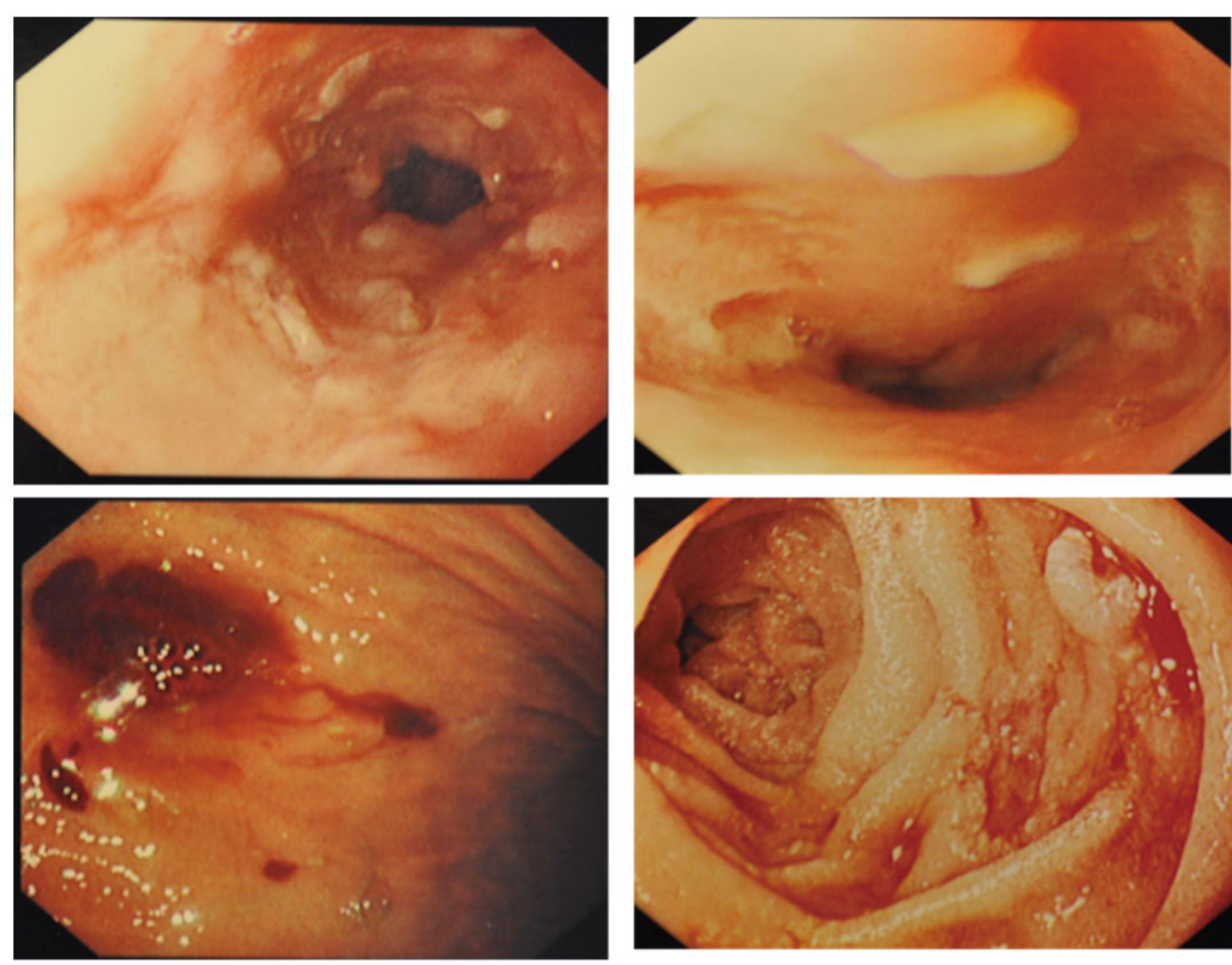

Figure 4. Esophagogastroduodenoscopy (EGD) showing multiple large ulcers from mid-esophagus to gastro-esophageal junction and distal duodenum

Mr. X was first started on oral prednisolone $1 \mathrm{mg} / \mathrm{kg} / \mathrm{day}$ and pulsed intravenous cyclophosphamide. Two days later, he complained of acute epigastric pain associated with tenderness and a significant drop in his hemoglobin. He initially declined an esophageal gastro-duodenoscopy (EGD) but agreed to it when he developed further epigastric pain and a $2 \mathrm{~g} / \mathrm{dl}$ drop in his hemoglobin level to $6.6 \mathrm{~g} / \mathrm{dl}$. Intravenous esomeprazole bolus was given followed by infusion. An emergent EGD showed multiple large ulcers from the midesophagus to the gastro-esophageal junction. A large amount of fresh blood was seen in the distal duodenum, obscuring the view of distal structures (see Figure 4).

Despite an initial injection of adrenaline, complete hemostasis was unable to be achieved. The procedure was then put on hold, and a repeat same-day EGD was resumed with a smaller-sized pediatric colonscope under monitored anesthesia care. The repeat EGD again showed multiple ulcers in the esophagus and stomach with no actively bleeding lesions. However, on advancement of the scope into the proximal jejunum, exposed vessel at the base of an ulcer was noted, and hemostasis was finally achieved by local injection of adrenaline and hemoclip application.

Mr. X was then transferred to the high-dependency unit and treated with continuous intravenous esomeprazole infusion.
His immunosuppressive therapy was withheld in view of the risk of exacerbating his GI bleeding. Unfortunately, despite initial seemingly promising progress, Mr. X would ultimately go on to develop acute massive melena two days after his scope, and succumbed to refractory hypovolemic shock.

\section{Discussion}

GPA, formerly known as Wegener's granulomatosis, is a systemic vasculitis of small-and medium-sized vessels characterized by necrotizing granulomatous inflammation that typically affects the respiratory tract and commonly involves the kidneys. GPA has an incidence of 5-10 cases per million population with males and females equally affected. ${ }^{[1]}$

According to American College of Rheumatology (ACR, 1990), GPA is defined by the presence of at least two of the four criteria: 1) nasal or oral inflammation, 2) abnormal chest radiograph with either the presence of nodules, fixed infiltrates or cavities, 3) urine sediment with hematuria or red cell cast, and 4) granulomatous inflammation on biopsy within an artery or in the perivascular area of an artery or arteriole. ${ }^{[3]}$ Our patient fulfilled the ACR criteria by having the evidence of pulmonary lesions and granulomatous inflammation seen in the skin, gum and kidney biopsies. The positive serum c-anti-neutrophilic cytoplasmic antibodies (ANCA) further support the diagnosis of GPA. 
GPA is a systemic disease that can affect any organs. Among the clinical manifestations, organs commonly affected include ear, nose and throat (70\%-100\%), lungs (50\%-90\%), kidneys (40\%-100\%), peripheral nervous system (6\%-13\%), skin $(10 \%-50 \%)$ and eyes $(14 \%-60 \%) .{ }^{[4]}$ Gastrointestinal involvement is notably rare, ranging from $0 \%-26 \%$ of cases in adults. ${ }^{[4-8]}$

The symptoms, onset and the severity of gastrointestinal involvement in patients with GPA vary. Symptoms that are previously reported in the literature include gingivitis, esophageal and gastric ulcers, small bowel perforation, colonic ulceration, non healing perianal ulcers, cholecystitis, pancreatitis, pancreatic mass and splenic necrosis. ${ }^{[9-15]}$

To date, gastrointestinal involvement in GPA is still poorly understood with only scattered case reports available. The most recent larger study to our knowledge is the study done by Masiak , the authors studied the clinical manifestations of gastrointestinal tract involvement in 9 out of 34 patients with GPA treated in their department. ${ }^{[16]}$ In order to further explore the clinical significance of this rare manifestation, we perform a literature review on cases reported on this subject from 1982 to June 2016.

The PubMed database from 1982 to June 2016 on GPA with gastrointestinal tract involvement in English literature was reviewed. A total of 49 case reports were explored. In addition to our case, the summary of the demographics and the clinicopathologic characteristics of these cases (a total of 50 cases) are shown in Table 1.

Table 1. Summary of cases of gastrointestinal involvement in patients with GPA in English literature from year 1982-June 2016

\begin{tabular}{|c|c|c|c|c|c|c|c|c|c|c|c|}
\hline Cases & $\begin{array}{l}\text { Age } \\
\text { (Yr) }\end{array}$ & Sex & GI site & Pathology & Biopsy & $\begin{array}{l}\text { Respi } / \text { /renal } \\
\text { involvement }\end{array}$ & $\begin{array}{l}\text { Onset of GI } \\
\text { symptoms }{ }^{\#}\end{array}$ & cANCA & $\begin{array}{l}\text { Therapy } \\
\text { prior }^{s}\end{array}$ & Surgery & Outcome \\
\hline $\begin{array}{l}\text { Spiera et al., } \\
1994^{[17]}\end{array}$ & 54 & $\mathrm{~F}$ & Esophagus & Erosions & $\begin{array}{l}\text { Necrotizing } \\
\text { granulomatous } \\
\text { inflammation }\end{array}$ & $\mathrm{Y} / \mathrm{Y}$ & 4 weeks & NS & $\mathrm{N}$ & $\mathrm{N}$ & Death \\
\hline $\begin{array}{l}\text { Fallows et al., } \\
2000^{[18]}\end{array}$ & 34 & $\mathrm{~F}$ & Esophagus & Ulcerations & $\begin{array}{l}\text { Inflammation, } \\
\text { fibrinoid necrosis }\end{array}$ & $\mathrm{N} / \mathrm{Y}$ & At onset & $+\mathrm{ve}$ & $\mathrm{N}$ & $\mathrm{N}$ & Survival \\
\hline $\begin{array}{l}\text { Matsumoto } \\
\text { et al., } 2007^{[19]}\end{array}$ & 72 & M & Esophagus & $\begin{array}{l}\text { Erosions, } \\
\text { ulcerations }\end{array}$ & Inflammation & $\mathrm{Y} / \mathrm{Y}$ & At onset & $+\mathrm{ve}$ & $\mathrm{N}$ & $\mathrm{N}$ & Survival \\
\hline $\begin{array}{l}\text { Yamaguchi } \\
\text { et al., } 2007^{[20]}\end{array}$ & 52 & $\mathrm{~F}$ & Esophagus & Stenosis & NS & $\mathrm{N} / \mathrm{N}$ & 2 months & $+\mathrm{ve}$ & $\mathrm{N}$ & $\mathrm{N}$ & Survival \\
\hline $\begin{array}{l}\text { Arista et al., } \\
2005^{[9]}\end{array}$ & 55 & M & Esophagus, stomach & Ulcerations & Ulcerations & $\mathrm{Y} / \mathrm{Y}$ & At onset & $+\mathrm{ve}$ & $\mathrm{N}$ & $\mathrm{N}$ & Survival \\
\hline $\begin{array}{l}\text { Deger et al., } \\
2008^{[21]}\end{array}$ & 34 & M & $\begin{array}{l}\text { Esophagus, stomach, } \\
\text { duodenum, jejunum }\end{array}$ & $\begin{array}{l}\text { Ulceration, } \\
\text { bleeding }\end{array}$ & $\begin{array}{l}\text { Inflammation, } \\
\text { fibrinoid necrosis, } \\
\text { vasculitis }\end{array}$ & $\mathrm{Y} / \mathrm{Y}$ & 3 months & $-\mathrm{ve}$ & $\mathrm{N}$ & $\mathrm{Y}$ & Survival \\
\hline $\begin{array}{l}\text { Alexander } \\
\text { et al., } 2015^{[22]}\end{array}$ & 56 & $\mathrm{~F}$ & Esophagus, ileum & $\begin{array}{l}\text { Ulcerations, } \\
\text { perforation }\end{array}$ & $\begin{array}{l}\text { Inflammation, } \\
\text { necrotic debris }\end{array}$ & $\mathrm{Y} / \mathrm{Y}$ & At onset & $+\mathrm{ve}$ & $\mathrm{N}$ & $\mathrm{N}$ & Death \\
\hline $\begin{array}{l}\text { Steele et al., } \\
2001^{[10]}\end{array}$ & 34 & $\mathrm{~F}$ & $\begin{array}{l}\text { Esophagus, stomach, } \\
\text { colon }\end{array}$ & $\begin{array}{l}\text { Ulcerations, } \\
\text { bleeding }\end{array}$ & Inflammation & $\mathrm{Y} / \mathrm{Y}$ & 3 weeks & $+\mathrm{ve}$ & $\mathrm{N}$ & $\mathrm{N}$ & Survival \\
\hline $\begin{array}{l}\text { Koçak et al., } \\
2012^{[23]}\end{array}$ & 39 & M & $\begin{array}{l}\text { Esophagus, duodenum, } \\
\text { colon }\end{array}$ & $\begin{array}{l}\text { Ulcerations, } \\
\text { bleeding }\end{array}$ & $\begin{array}{l}\text { Inflammation, } \\
\text { necrotizing } \\
\text { granulomas, vasculitis }\end{array}$ & $\mathrm{Y} / \mathrm{Y}$ & 2 months & $+\mathrm{ve}$ & $\mathrm{Y}$ & $\mathrm{Y}$ & Survival \\
\hline $\begin{array}{l}\text { Reddy et al., } \\
2006^{[11]}\end{array}$ & 34 & $\mathrm{~F}$ & $\begin{array}{l}\text { Esophagus, stomach, } \\
\text { duodenum, colon, } \\
\text { rectum }\end{array}$ & $\begin{array}{l}\text { Ulcerations, } \\
\text { strictures }\end{array}$ & Inflammation & $\mathrm{Y} / \mathrm{Y}$ & 10 weeks & $+\mathrm{ve}$ & $\mathrm{Y}$ & $\mathrm{N}$ & Survival \\
\hline Our case & 61 & M & $\begin{array}{l}\text { Esophagus, stomach, } \\
\text { duodenum }\end{array}$ & $\begin{array}{l}\text { Ulcerations, } \\
\text { bleeding }\end{array}$ & No biopsy taken & $\mathrm{Y} / \mathrm{Y}$ & 8 weeks & $+\mathrm{ve}$ & $\mathrm{Y}$ & $\mathrm{N}$ & Death \\
\hline $\begin{array}{l}\text { Yamauchi et al., } \\
1995^{[24]}\end{array}$ & 55 & M & Stomach & $\begin{array}{l}\text { Ulcerations, } \\
\text { bleeding }\end{array}$ & $\begin{array}{l}\text { Mononuclear cell } \\
\text { infiltration }\end{array}$ & $\mathrm{Y} / \mathrm{Y}$ & 1 month & $+\mathrm{ve}$ & $\mathrm{Y}$ & $\mathrm{N}$ & Survival \\
\hline $\begin{array}{l}\text { Zheng et al., } \\
2015^{[7]}\end{array}$ & 31 & $\mathrm{~F}$ & Stomach & Ulcerations & $\begin{array}{l}\text { Chronic } \\
\text { inflammation, } \\
\text { granulomas }\end{array}$ & $\mathrm{N} / \mathrm{Y}$ & At onset & $+\mathrm{ve}$ & $\mathrm{N}$ & $\mathrm{N}$ & Survival \\
\hline $\begin{array}{l}\text { Shahedi et al., } \\
2013^{[25]}\end{array}$ & 57 & M & $\begin{array}{l}\text { Stomach, duodenum, } \\
\text { jejunum }\end{array}$ & Inflammation & Inflammation & $\mathrm{Y} / \mathrm{Y}$ & At onset & $+\mathrm{ve}$ & $\mathrm{N}$ & $\mathrm{N}$ & Survival \\
\hline $\begin{array}{l}\text { Malik et al., } \\
2015^{[26]}\end{array}$ & 48 & M & Stomach & Ulcerations & $\begin{array}{l}\text { Chronic } \\
\text { inflammation, } \\
\text { non-necrotizing } \\
\text { granulomatous }\end{array}$ & $\mathrm{Y} / \mathrm{N}$ & At onset & $+\mathrm{ve}$ & $\mathrm{N}$ & $\mathrm{N}$ & Survival \\
\hline $\begin{array}{l}\text { Marie et al., } \\
2010^{[28]}\end{array}$ & 31 & M & $\begin{array}{l}\text { Duodenum, sigmoid, } \\
\text { rectum }\end{array}$ & $\begin{array}{l}\text { Erosions, } \\
\text { ulcerations }\end{array}$ & $\begin{array}{l}\text { Inflammation } \\
\text { surrounding blood } \\
\text { vessel }\end{array}$ & $\mathrm{N} / \mathrm{N}$ & At onset & $+\mathrm{ve}$ & $\mathrm{N}$ & $\mathrm{N}$ & Survival \\
\hline $\begin{array}{l}\text { Samim et al., } \\
2010^{[6]}\end{array}$ & 35 & M & Proximal jejunum & $\begin{array}{l}\text { Perforation, } \\
\text { bleeding }\end{array}$ & $\begin{array}{l}\text { Inflammation, } \\
\text { ulcerations }\end{array}$ & $\mathrm{Y} / \mathrm{Y}$ & 4 months & $+\mathrm{ve}$ & $\mathrm{Y}$ & $\mathrm{Y}$ & Survival \\
\hline $\begin{array}{l}\text { Skaife et al., } \\
2000^{[12]}\end{array}$ & 69 & M & Distal jejunum & Perforation & Vasculitis & $\mathrm{Y} / \mathrm{Y}$ & At onset & $+\mathrm{ve}$ & $\mathrm{N}$ & $\mathrm{Y}$ & Death \\
\hline
\end{tabular}

(Table 1 continued on page 38) 


\begin{tabular}{|c|c|c|c|c|c|c|c|c|c|c|c|}
\hline Cases & $\begin{array}{l}\text { Age } \\
\text { (Yr) }\end{array}$ & Sex & GI site & Pathology & Biopsy & $\begin{array}{l}\text { Respi }{ }^{*} / \text { renal } \\
\text { involvement }\end{array}$ & $\begin{array}{l}\text { Onset of GI } \\
\text { symptoms }^{\#}\end{array}$ & cANCA & $\begin{array}{l}\text { Therapy } \\
\text { prior }^{\$}\end{array}$ & Surgery & Outcome \\
\hline $\begin{array}{l}\text { Veinot et al., } \\
2003^{[29]}\end{array}$ & 71 & $\mathrm{~F}$ & Jejunum, ileum & $\begin{array}{l}\text { Ischemia, } \\
\text { necrosis }\end{array}$ & $\begin{array}{l}\text { Ulcerations, necrosis, } \\
\text { vasculitis }\end{array}$ & $\mathrm{Y} / \mathrm{Y}$ & 2 years & $+\mathrm{ve}$ & $\mathrm{Y}$ & $\mathrm{Y}$ & Survival \\
\hline $\begin{array}{l}\text { Shaikh et al., } \\
2006^{[13]}\end{array}$ & 44 & $\mathrm{~F}$ & $\begin{array}{l}\text { Distal jejunum, } \\
\text { ileum, colon }\end{array}$ & Perforation & Vasculitis, necrosis & $\mathrm{N} / \mathrm{N}$ & 8 weeks & $+\mathrm{ve}$ & $\mathrm{Y}$ & $\mathrm{Y}(>1)$ & Survival \\
\hline $\begin{array}{l}\text { Bulus et al., } \\
2013^{[30]}\end{array}$ & 47 & $\mathrm{~F}$ & Jejunum, ileum & $\begin{array}{l}\text { Necrosis, } \\
\text { perforation }\end{array}$ & $\begin{array}{l}\text { Necrotizing granulomatous } \\
\text { vasculitis }\end{array}$ & $\mathrm{Y} / \mathrm{Y}$ & At onset & $+\mathrm{ve}$ & $\mathrm{N}$ & $\mathrm{Y}$ & Death \\
\hline $\begin{array}{l}\text { Mcnabb et al., } \\
1982^{[31]}\end{array}$ & 50 & M & Distal ileum & $\begin{array}{l}\text { Ulcerations, } \\
\text { perforation }\end{array}$ & Nonspecific inflammation & $\mathrm{Y} / \mathrm{Y}$ & 9 months & NS & $\mathrm{Y}$ & $\mathrm{Y}$ & Survival \\
\hline $\begin{array}{l}\text { Coward et al., } \\
1985^{[32]}\end{array}$ & 46 & M & Distal ileum & $\begin{array}{l}\text { Ulcerations, } \\
\text { bleeding }\end{array}$ & Vasculitis & $\mathrm{Y} / \mathrm{Y}$ & 6 months & NS & $\mathrm{Y}$ & $\mathrm{Y}(>1)$ & Survival \\
\hline $\begin{array}{l}\text { Geraghty et al., } \\
1986^{[33]}\end{array}$ & 46 & M & $\begin{array}{l}\text { Distal ileum, } \\
\text { colon }\end{array}$ & $\begin{array}{l}\text { Ulcerations, } \\
\text { perforation }\end{array}$ & NS & $\mathrm{Y} / \mathrm{Y}$ & 4 weeks & NS & $\mathrm{Y}$ & $\mathrm{Y}$ & Death \\
\hline $\begin{array}{l}\text { Tokuda et al., } \\
1989^{[34]}\end{array}$ & 37 & M & Distal ileum & Perforation & Vasculitis & $\mathrm{Y} / \mathrm{Y}$ & 2 years & NS & $\mathrm{Y}$ & $\mathrm{Y}$ & Survival \\
\hline $\begin{array}{l}\text { Tupler et al., } \\
1991^{[35]}\end{array}$ & 55 & $\mathrm{~F}$ & $\begin{array}{l}\text { Distal ileum, } \\
\text { cecum }\end{array}$ & $\begin{array}{l}\text { Necrosis, } \\
\text { perforation }\end{array}$ & $\begin{array}{l}\text { Necrotizing granulomas, } \\
\text { vasculitis }\end{array}$ & $\mathrm{Y} / \mathrm{Y}$ & 1 year & NS & NS & $\mathrm{Y}$ & Death \\
\hline $\begin{array}{l}\text { Izzedine et al., } \\
2001^{[36]}\end{array}$ & 45 & M & Distal ileum & Ulcerations & Inflammation & $\mathrm{Y} / \mathrm{Y}$ & 9 years & $+\mathrm{ve}$ & $\mathrm{N}$ & $\mathrm{N}$ & Survival \\
\hline $\begin{array}{l}\text { Kitamura et al., } \\
2004^{[37]}\end{array}$ & 32 & M & $\begin{array}{l}\text { Distal ileum, asc. } \\
\text { colon }\end{array}$ & $\begin{array}{l}\text { Ulcerations, } \\
\text { bleeding }\end{array}$ & $\begin{array}{l}\text { Nonspecific inflammation, } \\
\text { vasculitis }\end{array}$ & $\mathrm{Y} / \mathrm{Y}$ & 9 months & $+\mathrm{ve}$ & $\mathrm{N}$ & $\mathrm{N}$ & Survival \\
\hline $\begin{array}{l}\text { Akça et al., } \\
2005^{[38]}\end{array}$ & 56 & M & Distal ileum & $\begin{array}{l}\text { Necrosis, } \\
\text { perforation }\end{array}$ & $\begin{array}{l}\text { Ulcerations, inflammation, } \\
\text { fistula, fibrosis }\end{array}$ & $\mathrm{Y} / \mathrm{N}$ & 6 months & $+\mathrm{ve}$ & $\mathrm{Y}$ & $\mathrm{Y}$ & Survival \\
\hline $\begin{array}{l}\text { Macías et al., } \\
2005^{[39]}\end{array}$ & 28 & NS & $\begin{array}{l}\text { Distal ileum, } \\
\text { colon }\end{array}$ & $\begin{array}{l}\text { Bowel wall } \\
\text { thickening }\end{array}$ & Vasculitis, granulomas & $\mathrm{Y} / \mathrm{N}$ & NS & $-\mathrm{ve}$ & NS & $\mathrm{Y}$ & Death \\
\hline $\begin{array}{l}\text { Strivens et al., } \\
2005^{[40]}\end{array}$ & 54 & $\mathrm{~F}$ & $\begin{array}{l}\text { Distal ileum, } \\
\text { colon }\end{array}$ & Perforation & Vasculitis & $\mathrm{Y} / \mathrm{Y}$ & 6 weeks & $+\mathrm{ve}$ & NS & $\mathrm{Y}$ & Survival \\
\hline $\begin{array}{l}\text { Kuwahara et al., } \\
2006^{[41]}\end{array}$ & 30 & M & $\begin{array}{l}\text { Distal ileum to } \\
\text { rectum }\end{array}$ & Ulcerations & Inflammation, granulomas & $\mathrm{Y} / \mathrm{Y}$ & 2 months & $+\mathrm{ve}$ & $\mathrm{N}$ & $\mathrm{N}$ & Survival \\
\hline $\begin{array}{l}\text { Beppu et al., } \\
2011^{[42]}\end{array}$ & 33 & M & $\begin{array}{l}\text { Distal ileum, } \\
\text { trans. colon, } \\
\text { cecum }\end{array}$ & Ulcerations & Inflammation, fibrosis & $\mathrm{Y} / \mathrm{Y}$ & 1 year & $+\mathrm{ve}$ & $\mathrm{Y}$ & $\mathrm{N}$ & Survival \\
\hline $\begin{array}{l}\text { Dag et al., } \\
2013^{[43]}\end{array}$ & 29 & M & $\begin{array}{l}\text { Distal ileum, } \\
\text { cecum, asc. } \\
\text { colon, hepatic } \\
\text { flexure }\end{array}$ & $\begin{array}{l}\text { Ulcerations, } \\
\text { bleeding }\end{array}$ & Nonspecific inflammation & $\mathrm{Y} / \mathrm{Y}$ & 6 months & $+\mathrm{ve}$ & $\mathrm{N}$ & $\mathrm{Y}$ & Survival \\
\hline $\begin{array}{l}\text { Deniz et al., } \\
2007^{[44]}\end{array}$ & 44 & M & Ileum & Perforation & $\begin{array}{l}\text { Ulcerations, necrotizing } \\
\text { granulomas, vasculitis }\end{array}$ & $\mathrm{Y} / \mathrm{N}$ & 1 month & $+\mathrm{ve}$ & NS & $\mathrm{Y}$ & Survival \\
\hline $\begin{array}{l}\text { Yildirim et al., } \\
2010^{[45]}\end{array}$ & 32 & M & Ileum & Perforation & $\begin{array}{l}\text { Inflammation, necrotizing } \\
\text { granulomatous vasculitis }\end{array}$ & $\mathrm{Y} / \mathrm{N}$ & 2 weeks & $+\mathrm{ve}$ & $\mathrm{Y}$ & $\mathrm{Y}$ & Death \\
\hline $\begin{array}{l}\text { Akbulut et al., } \\
2012^{[5]}\end{array}$ & 47 & M & Ileum & $\begin{array}{l}\text { Perforation, } \\
\text { fistula }\end{array}$ & NS & $\mathrm{Y} / \mathrm{Y}$ & 1.5 year & NS & $\mathrm{Y}$ & $\mathrm{Y}$ & Death \\
\hline $\begin{array}{l}\text { Srinivasan et al., } \\
1999^{[46]}\end{array}$ & 56 & $\mathrm{~F}$ & Small bowel & Perforation & Granulomatous reaction & $\mathrm{N} / \mathrm{N}$ & 10 weeks & $+\mathrm{ve}$ & $\mathrm{Y}$ & $\mathrm{Y}$ & Survival \\
\hline $\begin{array}{l}\text { Chow et al., } \\
2003^{[47]}\end{array}$ & 46 & M & Small bowel & $\begin{array}{l}\text { Ulcerations, } \\
\text { bleeding }\end{array}$ & Vasculitis & $\mathrm{Y} / \mathrm{Y}$ & 5 weeks & $+\mathrm{ve}$ & $\mathrm{Y}$ & $\mathrm{Y}(>1)$ & Survival \\
\hline $\begin{array}{l}\text { Dinić et al., } 2013 \\
{[48]}\end{array}$ & 52 & $\mathrm{~F}$ & Small bowel & Perforation & NS & $\mathrm{Y} / \mathrm{Y}$ & 10 months & $+\mathrm{ve}$ & $\mathrm{N}$ & $\mathrm{Y}$ & Death \\
\hline $\begin{array}{l}\text { Schneider et al., } \\
1997^{[14]}\end{array}$ & 41 & M & Colon & Ulcerations & Ulcerating colitis & $\mathrm{Y} / \mathrm{Y}$ & 3 years & $+\mathrm{ve}$ & $\mathrm{N}$ & $\mathrm{N}$ & Survival \\
\hline $\begin{array}{l}\text { Qian et al., } \\
2010^{[49]}\end{array}$ & 79 & $\mathrm{~F}$ & Colon & $\begin{array}{l}\text { Ulcerations, } \\
\text { bleeding }\end{array}$ & Inflammation, ulcerations & $\mathrm{Y} / \mathrm{Y}$ & At onset & $+\mathrm{ve}$ & $\mathrm{N}$ & $\mathrm{N}$ & Survival \\
\hline $\begin{array}{l}\text { Morchón Simón } \\
\text { et al., 2011 [50] }\end{array}$ & 43 & M & Colon & Inflammation & Inflammation & $\mathrm{Y} / \mathrm{Y}$ & At onset & $+\mathrm{ve}$ & $\mathrm{N}$ & $\mathrm{N}$ & Survival \\
\hline $\begin{array}{l}\text { Yoshikawa et al., } \\
2015^{[51]}\end{array}$ & 30 & M & Colon & $\begin{array}{l}\text { Inflammation } \\
\text {, bleeding }\end{array}$ & Inflammation & $\mathrm{N} / \mathrm{N}$ & At onset & $+\mathrm{ve}$ & $\mathrm{N}$ & $\mathrm{N}$ & Survival \\
\hline $\begin{array}{l}\text { Srivastava et al., } \\
2014^{[52]}\end{array}$ & 45 & $\mathrm{~F}$ & Colon & Inflammation & Vasculitis & $\mathrm{Y} / \mathrm{Y}$ & 1 week & $+\mathrm{ve}$ & $\mathrm{Y}$ & $\mathrm{Y}$ & Survival \\
\hline $\begin{array}{l}\text { Storesund et al., } \\
1998^{[53]} \text { Case } 1\end{array}$ & 26 & M & Sigmoid & Perforation & Vasculitis & $\mathrm{Y} / \mathrm{Y}$ & 18 months & $+\mathrm{ve}$ & $\mathrm{Y}$ & $\mathrm{Y}$ & Survival \\
\hline $\begin{array}{l}\text { Storesund et al., } \\
1998^{[53]}\end{array}$ & 46 & $\mathrm{~F}$ & $\begin{array}{l}\text { Sigmoid } \\
\text { Small bowel, }\end{array}$ & Inflammation & Vasculitis & $\mathrm{Y} / \mathrm{Y}$ & 10 months & $+\mathrm{ve}$ & $\mathrm{Y}$ & $\mathrm{Y}$ & Survival \\
\hline Case $2^{\&}$ & 55 & $\mathrm{~F}$ & colon & Necrosis & Ischemia & & 11 years & & $\mathrm{Y}$ & $\mathrm{Y}$ & Survival \\
\hline $\begin{array}{l}\text { Haworth et al., } \\
1984^{[54]}\end{array}$ & 43 & $\mathrm{~F}$ & Rectum & Ulcerations & Neutrophils & $\mathrm{Y} / \mathrm{Y}$ & 11 months & NS & $\mathrm{N}$ & $\mathrm{N}$ & Survival \\
\hline $\begin{array}{l}\text { Sinnott et al., } \\
2013^{[55]}\end{array}$ & 29 & M & Rectum & Inflammation & Inflammation & $\mathrm{Y} / \mathrm{Y}$ & At onset & $+\mathrm{ve}$ & $\mathrm{N}$ & $\mathrm{N}$ & Survival \\
\hline
\end{tabular}

In contrast to the equal frequency of GPA in males and fe- times commoner in males. ${ }^{[8]}$ The age of onset of the cases males in larger clinical studies, our literature review shows more males (31 patients) to females (18 patients) presented with gastrointestinal manifestations in GPA. ${ }^{[56,57]}$ This is, however similar to a smaller study where GPA was about 1.5 reported in our review ranges from 26 to 79 year.

Out of the total 50 case reports, 38 patients had classical GPA involving both the lungs and kidneys while only 5 
patients presented unusually without either. Out of the 42 case reports with c-ANCA status documented, 40 cases were positive. This is consistent with the previous study which suggested most patients (approximately 90\%) with active, generalized GPA had positive serum ANCA. ${ }^{[58]}$

Overall, GPA can affect any part of the gastrointestinal tract. In fact, most of the cases reported involved multiple locations. Of note, small bowel had the highest frequency with 33 cases while rectum involvement was only reported in 2 cases. Esophageal and gastric manifestations were reported in 9 and 11 cases respectively.

Inflammation and ulcerations were the most frequently seen pathology in endoscopy or laparotomy and among all the cases reported, 17 patients suffered from gastrointestinal perforation. Microscopically, most cases showed non-specific inflammation and ulceration. These frequent non-specific inflammation found in biopsies is not uncommon and was previously suggested by Camilleri et al. that it might be a result of biopsy taken too superficially. ${ }^{[59]}$ The difficulty in demonstrating the presence of granulomatous inflammation/vasculitis from gastrointestinal tract mucosa places a challenge to differentiate GPA from inflammatory bowel disease. ${ }^{[14,25]}$

In all patients, only 15 cases had gastrointestinal symptoms appeared at the onset of the disease. The gastrointestinal involvement in GPA occurred from 1 week to 11 years. This is in contrast to Masiak et al.'s study where all their patients (9 out of 34) manifested gastrointestinal symptoms within the first year with the most common symptoms being abdominal pain and gastrointestinal bleeding. ${ }^{[16]}$

As gastrointestinal involvement in GPA is rare, the use of immunosuppressive therapy especially corticosteroid has been speculated to be the possible cause to the development of gastrointestinal manifestations in GPA. ${ }^{[16,53]}$ In our literature review, a total of 21 patients developed gastrointestinal symptoms after immunosuppressive therapy. Of the cases with known duration of therapy prior to the gastrointestinal manifestations, the period of time ranges from 1 day to 12 months. 11 cases detected vasculitis/granulomatous inflammation histopathologically. Most of these cases (16 patients) improved and survived. Furthermore, Izzedine $e t$ al.'s patient presented with gastrointestinal manifestation as a relapsing symptom of GPA. ${ }^{[36]}$ These results suggest

\section{REFERENCES}

[1] Lutalo PM, D'Cruz DP. Diagnosis and classification of granulomatosis with polyangiitis (aka Wegener's granulomatosis). Journal of Autoimmunity. 2014; 48: 94-8. PMid:24485158 https:

Published by Sciedu Press that the gastrointestinal tract involvement is likely a result from the disease process of GPA itself rather than due to the immunosuppressive agents.

Interestingly, out of the 25 cases that required surgery, 3 patients had more than 1 operation. In fact, some of the patients described in the case reports required multiple endoscopies to localize and control the source of bleeding. This may suggest that gastrointestinal manifestations in GPA can be progressive and/or recurrent.

GPA is the most common form of life-threatening smallvessel vasculitis, however, death from gastrointestinal complication is extremely rare. Luqmani et al. identified 255 patients with GPA and long-term mortality in patients with GPA was compared with matched population-based controls, there was only 1 patient died from bowel perforation in within a year. Most of the causes of death were due to infection, disease activity and renal failure. ${ }^{[2]}$

In our literature, a total of 12 patients passed away despite treatment. As most of the cases reported involved multiple organs, the higher mortality rate in our review (24\%) may likely be that the gastrointestinal manifestations occur during the acute phase of the disease and death occurs due to multi-systemic involvement. It is unsure whether gastrointestinal involvement could indicate a poor prognostic factor given its rare occurrence in GPA. However, given the high mortality rate and the possible catastrophic gastrointestinal hemorrhagic and perforation incidents in such manifestation, a high clinical suspicion, early treatment and close surveillance upon initiation of immunosuppressive agents are warranted.

\section{Conclusions}

Although uncommon, massive GI ulcerative bleeding can occur in GPA as a result of the underlying disease and/or aggravated by concurrent immunosuppressive therapy. This can be potentially catastrophic and warrants a high index of suspicion and close monitoring.

\section{ETHICS}

Written informed consent was obtained from the patient's next of kin for publication of this case report and accompanying images.

\section{CONFlicts of InTEREST Disclosure}

The authors have no competing interests to declare.
] Luqmani R, Suppiah R, Edwards CJ, et al. Mortality in Wegener's granulomatosis: A bimodal pattern. Rheumatology. 2011; 50(4): 697-702. 
[3] Leavitt RY, Fauci AS, Bloch DA, et al. The American College of Rheumatology 1990 criteria for the classification of Wegener's granulomatosis. Arthritis \& Rheumatism. 1990; 33(8): 1101-7. https://doi.org/10.1002/art.1780330807

[4] Comarmond C, Cacoub P. Granulomatosis with polyangiitis (Wegener): clinical aspects and treatment. Autoimmunity Reviews. 2014; 13(11): 1121-5. PMid:25149391 https://doi.org/10.1016/j. autrev.2014.08.017

[5] Akbulut S. Multiple ileal perforations in a patient with Wegener's granulomatosis: A case report and literature review. Journal of Gastrointestinal Surgery. 2012; 16(4): 857-62. PMid:22042563 https://doi.org/10.1007/s11605-011-1735-z

[6] Samim M, Pronk A, Verheijen PM. Citation of This Article. World 2010; 2(5): 169-71.

[7] Zheng Z, Ding J, Li X, et al. Gastric presentation (vasculitis) mimics a gastric cancer as initial symptom in granulomatosis with polyangiitis: A case report and review of the literature. Rheumatology International. 2015; 35(11): 1925-9. PMid:26248531 https : //doi.org/10.1007/s00296-015-3334-x

[8] Martínez-Morillo M, Grados D, Naranjo-Hans D, et al. Granulomatosis con poliangeítis (Wegener). Description of 15 cases. Reumatología Clínica. 2012; 8(1): 15-9. PMid:22089070 https ://doi .org/10 .1016/j.reuma.2011.04.009et al.

[9] Arista S, Sailler L, Astudillo L. Relapsing esophageal and gastric ulcers revealing Wegener's granulomatosis. The American Journal of Medicine. 2005; 118(8): 923-4. PMid:16084188 https: //doi.org/10.1016/j.amjmed.2004.12.032

[10] Steele C, Bohra S, Broe P, et al. Acute upper gastrointestinal haemorrhage and colitis: An unusual presentation of Wegener's granulomatosis. European Journal of Gastroenterology \& Hepatology. 2001; 13(8): 993-5. https ://doi.org/10.1097/00042737-2001080 00-00023

[11] Reddy RS, Biyyani S, Pauskar P, et al. Extensive gastrointestinal tract and thyroid involvement with Wegeners granulomatosis. Indian Journal of Gastroenterology: Official Journal of the Indian Society of Gastroenterology. 2006; 26(6): 290-1.

[12] Skaife P, Lee S, Maitra D, et al. Intestinal perforation as a presentation of Wegener's granulomatosis. Hospital Medicine. 2000; 61(4): 2867. PMid:10858811 https://doi .org/10.12968/hosp. 2000.6 1.4 .1320

[13] Shaikh F, Sabu C, Peirce T, et al. Extensive intestinal ischaemic necrosis in Wegener's granulomatosis. Gut. 2006; 55(9): 1368-9. PMid:16905710 https ://doi.org/10.1136/gut.2006.09676 8

[14] Schneider A, Menzel J, Gaubitz M, et al. Colitis as the initital presentation of Wegener's granulomatosis. Journal of Internal Medicine. 1997; 242(6): 513-7. PMid:9437413 https://doi.org/10.111 1/j.1365-2796.1997.tb00025.x

[15] Cojocaru M, Cojocaru IM, Silosi I, et al. Gastrointestinal manifestations in systemic autoimmune diseases. Maedica-a Journal of Clinical Medicine. 2011; 6(1).

[16] Masiak A, Zdrojewski L, Zdrojewski Z, et al. Gastrointestinal tract involvement in granulomatosis with polyangiitis. Przeglad Gastroenterologiczny. 2016; 11(4): 270-5.

[17] Spiera RF, Filippa DA, Bains MS, et al. Esophageal involvement in Wegener's granulomatosis. Arthritis \& Rheumatism. 1994; 37(9): 1404-7. https://doi .org/10.1002/art. 1780370920

[18] Fallows GA, Hamilton SF, Taylor DS, et al. Esophageal involvement in Wegener's granulomatosis: A case report and review of the literature. Canadian Journal of Gastroenterology and Hepatology. 2000; 14(5): 449-51. https://doi.org/10.1155/2000/423569
[19] Matsumoto M, Nakamura T, Ohashi T, et al. Esophageal involvement in microscopic polyangiitis: A case report and review of literature. Internal Medicine. 2007; 46(10): 663-7. PMid:17527040 https://doi.org/10.2169/internalmedicine.46.6115

[20] Yamaguchi T, Yoshioka K, Ueda M, et al. A case of Wegener's granulomatosis associated with progressive dysphagia owing to esophageal involvement. Modern Rheumatology. 2007; 17(6): 521-5. PMid:18084709 https : //doi.org/10.3109/s10165-007-063 3-4

[21] Deger SM, Sahin T, Vural C, et al. Wegener's granulomatosis with massive gastrointestinal hemorrhage due to jejunal and colonic involvement: report of a case. Surgery Today. 2008; 38(2): 166-9. https://doi.org/10.1007/s00595-007-3574-9

[22] Alexander S, Simon EG, Burad D, et al. Esophageal vasculitis in granulomatosis with polyangiitis. Tropical Gastroenterology. 2015; 36(2): 132-4. PMid:26710488 https ://doi .org/10.7869/tg . 270

[23] Koçak G, Koçak E, Huddam B, et al. Successful Surgical Treatment of Massive Lower Gastrointestinal Bleeding in a Patient with Wegener's Granulomatosis. The American Surgeon. 2012; 78(9): E416. PMid:22964183

[24] Yamauchi F, Tanabe R, Nakashima R, et al. Wegener's granulomatosis with relapsed bleeding of gastric ulcers: A case report. Tokai Journal of Experimental and Clinical Medicine. 1995; 20(4): 233-40. PMid:8956466

[25] Shahedi K, Hanna RM, Melamed O, et al. Wegener's granulomatosis mimicking inflammatory bowel disease and presenting with chronic enteritis. International Medical Case Reports Journal. 2013; 6: 65. PMid:24124396

[26] Malik M, Ismail M, Pattanaik D. Granulomatosis With Polyangiitis Presenting as Gastric Ulcer: An Unusual Initial Manifestation Successfully Treated With Rituximab. The American Journal of the Medical Sciences. 2015; 350(4): 338-9. PMid:26191640 https ://doi.org/10.1097/MAJ .0000000000000536

[27] Arhan M, Köklü S, Yalçin F, et al. Severe Intestinal Bleeding in a Patient With Wegener's Granulomatosis. The American Journal of Gastroenterology. 2009; 104(8): 2119. PMid:19455112 https : //doi.org/10.1038/ajg.2009. 209

[28] Marie I, Lahaxe L, Josse S, et al. Rectal involvement revealing Wegener's disease. Scandinavian Journal of Rheumatology. 2010; 39(4): 346-8. PMid:20476865 https ://doi.org/10.3109/0300 9740903524388

[29] Veinot J, Logan C, Thomas MJ. Wegener's granulomatosis arteritis causing small bowel infarction. Pathology. 2003; 35(3): 268-9. PMid:14506977 https://doi.org/10.1080/00313020310001 51046

[30] Bulus H, Koçak E, Yavuz A, et al. Intestinal perforation as the initial presentation of Wegener's granulomatosis. Rheumatology International. 2013: 1-2. https://doi.org/10.1007/s00296-012-2 517-y

[31] McNabb W, Lennox M, Wedzicha J. Small intestinal perforation in Wegener's granulomatosis. Postgraduate Medical Journal. 1982; 58(676): 123-5.

[32] Coward R, Gibbons C, Brown C, et al. Gastrointestinal haemorrhage complicating Wegener's granulomatosis. British Medical Journal. 1985; 291(6499): 865. https://doi.org/10.1136/bm j . 291.64 99.865

[33] Geraghty J, Mackay I, Smith D. Intestinal perforation in Wegener's granulomatosis. Gut. 1986; 27(4): 450-1. PMid:3957113 https : //doi.org/10.1136/gut.27.4.450

[34] Tokuda M, Kurata N, Daikuhara H, et al. Small intestinal perforation in Wegener's granulomatosis. The Journal of Rheumatology. 1989; 16(4): 547-9. PMid:2746597 
[35] Tupler RH, McCuskey WH. Wegener granulomatosis of the colon: CT and histologic correlation. Journal of Computer Assisted Tomography. 1991; 15(2): 314-6. https://doi.org/10.1097/000047 28-199103000-00025

[36] Izzedine H, Lacaille S, Deray G. An unusual presentation of relapsing Wegener's granulomatosis. Age (years). 2001; 20(26A55): 45.

[37] Kitamura N, Matsukawa Y, Takei M, et al. Wegener's granulomatosis complicated with intestinal ulceration. Modern Rheumatology. 2004; 14(6): 480-4. PMid:24387727 https://doi.org/10.3109/s101 65-004-0343-0

[38] Akça T, Çolak T, Caglikulekci M, et al. Intestinal perforation in Wegener's granulomatosis: A case report. Ulusal Travma ve Acil Cerrahi Dergisi. 2005; 11(4): 348. PMid:16341975

[39] Macías MS, Bernal MS, Artacho GS, et al. Severe intestinal involvement in Wegener's granulomatosis with negative c-ANCAs. Revista Espanola de Enfermedades Digestivas. 2005; 97(9): 670. https://doi.org/10.4321/s1130-01082005000900008

[40] Strivens R, Bateman A, Arden N, et al. Intestinal perforation and jejunal haemorrhage due to Wegener's granulomatosis. Clinical and Experimental Rheumatology. 2005; 23(1): 124. PMid:15789904

[41] Kuwahara Y, Shima Y, Tanaka T, et al. Successful treatment with intravenous cyclophosphamide pulse therapy of severe intestinal involvement in Wegener's granulomatosis. Scandinavian Journal of Rheumatology. 2006; 35(3): 243-5. PMid:16766375 https: //doi.org/10.1080/03009740500381179

[42] Beppu K, Osada T, Inoue K, et al. Intestinal involvement in Wegener's granulomatosis diagnosed and followed up by Double Balloon Enteroscopy. Internal Medicine. 2011; 50(3): 219-22. PMid:21297323 https://doi.org/10.2169/internalmedicine.50.4188

[43] Dag MS, Pehlivan Y, Tutar E, et al. Rituximab seems a promising therapeutic option in granulomatosis with polyangiitis with intestinal perforation: A case report and literature review. BMJ Case Reports. 2013; 2013: bcr2012007518.

[44] Deniz K, Ozşeker H, Balas S, et al. Intestinal involvement in Wegener's granulomatosis. Journal of Gastrointestinal and Liver diseases: JGLD. 2007; 16(3): 329-31. PMid:17925931

[45] Yildirim A, Koçak E, Yildiz P, et al. Multiple intestinal perforation in a patient with Wegener's granulomatosis: A case report and review of the literature. Gastroentérologie Clinique et Biologique. 2010; 34(12): 712-5. PMid:20884146 https://doi .org/10.101 $6 / j \cdot g c b .2010 .08 .009$

[46] Srinivasan U, Coughlan R. Small intestinal perforation complicating Wegener's granulomatosis. Rheumatology. 1999; 38(3): 289-90. PMid:10325676 https://doi.org/10.1093/rheumatology/3 8.3.289
[47] Chow FY, Hooke D, Kerr PG. Severe intestinal involvement in Wegener's granulomatosis. Journal of Gastroenterology and Hepatology. 2003; 18(6): 749-50. PMid:12753164 https://doi.org/10.104 6/j.1440-1746.2003.30472.x

[48] Dinić MŽ, Kandolf-Sekulović L, Zolotarevski L, et al. Fulminant Wegener's granulomatosis: A case report. Vojnosanitetski pregled. 2013; 70(9): 887-90. PMid:24266321 https://doi.org/10.229 8/VSP130111023D

[49] Qian Q, Cornell L, Chandan V, et al. Hemorrhagic colitis as a presenting feature of Wegener granulomatosis. J Gastrointestin Liver Dis. 2010; 19(4): 445-7. PMid:21188339

[50] Morchón-Simón D, Martín-Escudero JC. Hemorrhagic colitis as the onset of Wegener's granulomatosis. International Journal of Colorectal Disease. 2011; 26(2): 259-60. PMid:20544207 https: //doi.org/10.1007/s00384-010-0977-7

[51] Yoshikawa A, Yoshida S, Takeuchi T, et al. Gastrointestinal involvement at the onset of granulomatosis with polyangiitis: A case report. Modern Rheumatology. 2015: 1-3. PMid:26474323

[52] Srivastava S, Devlin A, Winn LYN, et al. Wegener's granulomatosisan unusual case of colonic haemorrhage. QJM. 2014; 107(10): 841-3. PMid:22351898 https://doi.org/10.1093/qjmed/hcs026

[53] Storesund B, Gran J, Koldingsnes W. Severe intestinal involvement in Wegener's granulomatosis: Report of two cases and review of the literature. Rheumatology. 1998; 37(4): 387-90. https : //doi.org/10.1093/rheumatology/37.4.387

[54] Haworth S, Pusey C. Severe intestinal involvement in Wegener's granulomatosis. Gut. 1984; 25(11): 1296-300. PMid:6500368 https : //doi.org/10.1136/gut.25.11.1296

[55] Sinnott JD, Matthews P, Fletcher S. Colitis: An unusual presentation of Wegener's granulomatosis. BMJ Case Reports. 2013; 2013: bcr2012007566.

[56] Scott DG, Watts RA. Systemic vasculitis: epidemiology, classification and environmental factors. Annals of the Rheumatic Diseases. 2000; 59(3): 161-3. https : //doi.org/10.1136/ard.59.3.161

[57] Hoffman GS, Kerr GS, Leavitt RY, et al. Wegener granulomatosis: An analysis of 158 patients. Annals of Internal Medicine. 1992; 116(6): 488-98. PMid:1739240 https://doi.org/10.7326/00 03-4819-116-6-488

[58] Mubashir E, Ahmed MM, Hayat S, et al. Wegener granulomatosis: A case report and update. Southern Medical Journal. 2006; 99(9): 977-89. PMid:17004532 https ://doi .org/10.1097/01.smj.0 $000232210.04881 . \mathrm{d} 3$

[59] Camilleri M, Pusey C, Chadwick V, et al. Gastrointestinal manifestations of systemic vasculitis. QJM. 1983; 52(2): 141-9. PMid:6604292 